\title{
Design and Achievement of Wireless Data Transmission Module in Life-Detection Radar
}

\author{
Xiao Yu, Yan Wang, Yang Zhang, Teng Jiao, Hua Wang, Xijing Jing, Jianqi Wang \\ Faculty of Biomedical Engineering \\ The Fourth Military Medical University \\ Xi'an, 710032, China \\ yuxiao@fmmu.edu.cn
}

\begin{abstract}
In this paper, a wireless data transmission module applicable in life-detection radar has been designed and achieved. The system use serial A/D converter TLC2543 and serial Bluetooth to make wireless data transmission module based on 51 series SCM. The schematic diagrams of hardware circuit and software system of the wireless transmission module were designed and the wireless data transmission was achieved. The design is recited in detail. This module can be operated steadily and reliably and increases the detection flexibility of life-detection radar.
\end{abstract}

Keywords-Bluetooth; life-detection; radar; SCM; wireless

\section{INTRODUCTION}

Life-detection radar combines radar technology and biomedical engineering technology, could penetrate nonmetal mediums (brick walls, ruins) to detect vital signs such as respiration and movement [1]. The principle of lifedetection radar is based on the detection of the modulated part of a scattered wave which is generated by the respiratory movements of human beings [2][3]. Recently, the lifedetection radar has to face with more and more complicated environment with its increasing important role in antiterrorist operations, rescue operations after disasters and other tasks. However, it is impossible for the traditional cable data transmission to meet the data transmission under complicated environment, especially under the environment after disasters. Therefore, the wireless data transmission becomes more flexible and feasible. The wireless data transmission module with Single Chip Microcomputer (SCM) and serial Bluetooth was designed in this paper according to the problems of life-detection radar emerged during the practical application.

\section{GENERAL DESIGN}

The SCM is widely applied in modern industrial control and data acquisition system due to its low cost, flexible programming, convenience, strong timeliness and certain intelligence. However, the life-detection radar has to finish abundant data operation after the data acquisition, which is powerless for the SCM but the strength of PC lies in. Therefore, it is necessary to complement their advantages to have the SCM responsible for controlling the executive body and data acquisition as well as transmission, while make PC responsible for analyzing the collected data and abundant data operation as well as storage.
In view of the above reasons, this paper designed and achieved a wireless data transmission module based on the SCM and serial Bluetooth. Taking the full advantage of the simple structure, high reliability, operability in complicated environment and other characteristics of SCM and combined with the Bluetooth technology, this module achieved the communication between SCM and PC through serial communication, thus realizing the goal of wireless data transmission. The module can transmit data as far as $100 \mathrm{~m}$, especially appropriate for the wireless data transmission of radar life detector. The technical roadmap of the system was shown in Fig.1. [4].

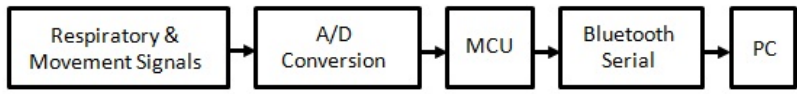

Figure 1. The technical roadmap of the system

Technical requirements of wireless data transmission module: (1) Input signal range: $\pm 5 \mathrm{~V}$. (2) A/D conversion resolution: 12 bit. (3) Processing chip: AT89S51. (4) Communication protocol: receive command and send the command initiatively. (5) Output data format: 12 hexadecimal data.

\section{HARDWARE DESIGN}

To meet the technical requirement of wireless data transmission module, AT89S51 was applied as core chip of this module and the power of SCM was supplied by lifedetection radar. The pre-processed respiration and body movement signals collected by the front end of radar were converted into digital signals by controlling the A/D converter through SCM, and the SCM controls the serial Bluetooth module after the level switch to transmit data to computer for follow-up processing in wireless manner.

\section{A. A/D Conversion}

It is primarily for the data transmission module to convert the transmitted analog signals into digital signals. Therefore, A/D conversion plays an important role. According to the technical requirement of the module, the TLC2543 made by TI Company was selected. TLC2543 is an 11 analog inputs, 12-bit switched capacity, successive approximation analogto-digital converter (A/D converter), which has various advantages, such as quick conversion speed, good stability, simple interface with 51 series SCM, etc. It can save I/O 
resources of SCM because of the serial input structure. Furthermore, it costs appropriately, but has higher resolution [5].

Since 51 series SCM has no serial peripheral equipment interface (SPI) or interfaces with same functions, it is necessary to choose clock frequency as high as possible for the convenience of regular operation of TLC2543 and reducing the impact of SCM's clock frequency on data transmission speed. When using TLC2543, the power end must be connected to the ground through a ceramic capacitor, serving as the decoupling capacitor. In the environment with huge noise impact, it is suggested to parallel connecting a capacitance between the power supply and ceramic capacitor, which could decrease the impact of noise. Necessary attentions shall be paid to ensure the digital signals separated from analog signals during the wiring of circuit board, ensuring that the analog line and digital line, especially the clock signal line cannot be parallel to each other, and the digital signal lines cannot be distributed under the TLC2543 chip.

\section{B. Level Switch}

Serial communication between the SCM and computer is convenient since the 51 series SCM has a full duplex serial communication port. However, a level switch circuit is necessary between the computer and the SCM, because the serial port of computer is RS232 level, while the serial port of SCM is TTL level. In this paper, the level switch chip MAX232 was selected and connection serial port of threewire system was applied. In other words, only 3 wires in the 9 pins of computer serial port were connected: GND of 5th pin, RXD of 2nd pin and TXD of 3rd pin.

\section{Serial Bluetooth Module}

In this paper, the wireless data transmission was achieved through serial Bluetooth module-BTS2502C1H made by Chongqing Jinou Company, as shown in Fig.2. It is a $100 \mathrm{~m}$ serial Bluetooth module, mainly responsible for converting the data collected by serial port of SCM into Bluetooth protocol to send to the Bluetooth equipment at the PC end, and converting the Bluetooth data packet received by the Bluetooth equipment at the PC end into serial data to send to the computer. Its maximum wireless communication distance can reach $100 \mathrm{~m}$ since its inbuilt power amplification module and antenna, completely capable to satisfy the practical application of radar life detector. The computer end applied the Bluetooth adapter with USB interface to achieve wireless data communication with serial Bluetooth module.

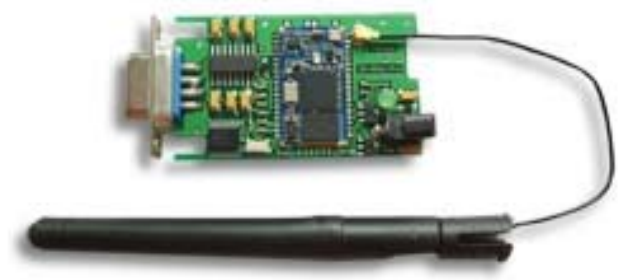

Figure 2. Serial Bluetooth Module

\section{Achievement of Serial Communication}

The basic operating principle of serial interface of 51 series SCM is: during sending, the parallel data sent by the SCM was converted into serial data with certain format, which will be sent one bit by one from TXD pin according to the specified Baud rate, while during receiving, the RXD pin was monitored and once the start bit emerged, the serial data with certain format sent by peripheral equipment will be converted into parallel data and wait for the read of SCM.

The serial port has four working modes, which were selected by Sm0 and SM1 in the SCON register. When $\mathrm{SM} 0=0$ and $\mathrm{SM} 1=1$, the serial port worked in mode 1 which is a 10-bit universal asynchronous interface used for serial data sending or receiving. Among them, TXD is responsible for sending data, while RXD is responsible for receiving data. One frame data includes a start bit, 8 data bits (from low bit to high bit) and a stop bit. The Baud rate of mode 1 is variable. This paper applied 38,400bps Baud rate and mode 1 as the full duplex serial port operation, that is, the 10-bit asynchronous send-receive manner controlled by timer. Therefore, the data transmission format is 10 bits, including 8 data bits, 1 stop bit " 1 " and 1 start bit " 0 ". The start bit and stop bit are inserted automatically during sending.

\section{SOFTWARE DESIGN}

The studied module was controlled by computer during wireless data transmission. The computer send command of data transmission and the module send data to the computer. Data of each channel occupied two bytes with higher byte in front and low byte behind. Among them, the high 4 bits in the high byte were the corresponding channel number, while the rest were corresponding to the 12-bit data converted by A/D. Therefore, the system software was composed of SCM control program, SCM data collection program and SCM data transmission program according to the required functions and modular design idea. 


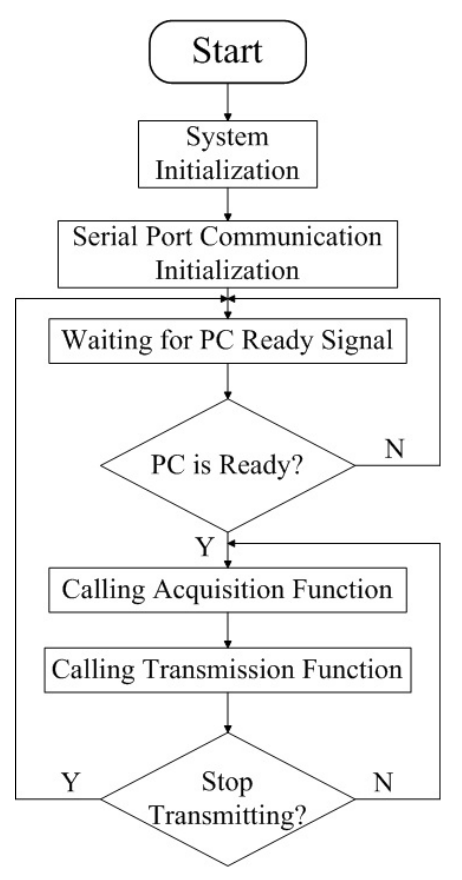

Figure 3. Software flow disgram of the system.

\section{A. SCM Control Program}

The SCM control program was mainly responsible for the operating mode setting of AT89S51 and TLC2543 A/D converter, initialization of serial communication and calling data collection subprogram as well as data transmission subprogram. Its software procedure was shown in Fig.3.

\section{B. SCM Data Acquisition Program}

The SCM data acquisition program mainly accomplishes 51 series SCM's control on TLC2543, including clock initialization, collection times setting, control word setting and data collection. The channel selection and mode data of TLC2543 is 8 bits, among which D7, D6, D5 and D4 are used to select the channels need conversion, while D3 and $\mathrm{D} 2$ are used to select the length of output data, which is 12 bits in this paper, and D1 and D0 select the leading position of input data

\section{SCM Data Transmission Program}

The SCM data transmission program mainly accomplishes the serial port initialization and data transmission. The serial port of SCM must be initialization programmed before the data transmission, because the serial communication between the SCM and computer requires same Baud rate and transmission bits in order to prevent communication confusion. The initialization programming mainly refers to set the timer producing Baud rate1 and set the communication mode of serial port.

\section{CONCLUSION}

The 51 series SCM and serial Bluetooth based wireless data transmission module was designed and achieved in this paper, which transmits the vital signs like breathe and body movement collected by radar life detector to the computer through wireless method for follow-up processing. This module extends the application range of radar life detector. In practical search and rescue, it overcomes the difficulties brought by the environmental complexity and enables the rescue workers to search and rescue far away from the radar and detection region. It provides support for the application of radar life detector in various complicated environment, thus possessing good practicability and application prospect.

\section{ACKNOWLEDGMENT}

This work was supported by Natural Science Foundation of Shaanxi Province under Grant 2012JM4047, by the National Natural Science Foundation of China under Grant 60927003.

\section{REFERENCES}

[1] S. I. Ivashov, V. V. Razevig, A.P. Sheyko, I. A. Vasilyev, "Detection of Human Breathing and Heartbeat by Remote Radar”, Progress in Electromagnetic Research Symposium 2004, Pisa, Italy, March 28-31, pp. 663-666.

[2] A. G. Yarovoy, L. P. Ligthart, J. Matuzas, B. Levitas, "UWB Radar for Human Being Detection”, IEEE Aerospace and Electronic Systems Magazine, vol. 21, no. 11, pp. 22-26, Nov, 2006.

[3] I. Immoreev, The-Ho Tao, "UWB radar for patient monitoring”, IEEE Aerospace and Electronic Systems Magazine, vol.23, no.11, pp.11-18, Nov. 2008

[4] AT89S51 Complete, Revision C, Atmel Co., San Jose, CA, 2008, pp. 1-31. Available: http://www.atmel.com/devices/at89s51.aspx

[5] TLC2543 Datasheet, Texas Instruments Co., 2001, pp. 1-33. Available: http://www.ti.com/lit/ds/symlink/tlc2543.pdf 